\title{
An electromagnetic cell-stretching device for mechanotransduction studies of olfactory ensheathing cells
}

\author{
Kamble Harshad 1 - Myeongjun Jun ${ }^{2} \cdot$ Sungsu Park ${ }^{2} \cdot$ Matthew J Barton $^{3} \cdot$ Raja K. Vadivelu $^{4}$. \\ James St John 4 - Nam-Trung Nguyen ${ }^{*}$
}

\begin{abstract}
Olfactory ensheathing cells (OECs) are primary candidates for cell transplantation therapy to repair spinal cord injury (SCI). However, the post transplantation survival of these cells remains a major hurdle for a success using this therapy. Mechanical stimuli may contribute to the maintenance of these cells and thus, mechanotransduction studies of OECs may serve as a key benefit to identify strategies for improvement in cell transplantation. We developed an electromagnetic cell stretching device based on a single sided uniaxial stretching approach to apply tensile strain to OECs in culture. This paper reports the design, simulation and characterisation of the stretching device with preliminary experimental observations of OECs in vitro. The strain field of the deformable membrane was investigated both experimentally and numerically. Heterogeneity of the device provided an ideal platform for establishing strain requirement for the OEC culture. The cell stretching system developed may serve as a tool in exploring the mechanobiology of OECs for future SCI transplantation research.
\end{abstract}

Keywords- Cell Stretching, Mechanotransduction, Biomedical Device, Mechanobiology.

*Email: nam-trung.nguyen@griffith.edu.au

1 Queensland Micro and Nanotechnology Center, Griffith University, Nathan, QLD, Australia.

2 School of Mechanical Engineering, Sungkyunkwan University, Suwon, Korea.

3 Menzies Health Institute Queensland, Griffith University, Gold Coast, QLD, Australia.

4 Eskitis Institute for Drug Discovery, Griffith University, Australia. 


\section{Introduction}

Cells in the human body are under constant influence from the various mechanical forces place upon them. The conversion of mechanical stimuli such as rigidity and stretching into intracellular biomolecular signaling is known as a mechanotransduction (Silver and Siperko, 2003; Brandes et al., 2014). Mechanical force is well known to be one of the most significant stimuli to influence cell growth (Cui et al., 2015), behaviour and morphology (Wang J. H. C. and Thampatty, 2006). Furthermore, abnormalities in mechanotransduction have been reported to lead to diseases such as asthma, heart failure and cancer (Ingber, 2003; DuFort et al., 2011). Thus, the development of techniques to best mimic the cells' physical microenvironment has been a major interest within the research community of mechanotransduction.

Nevertheless, the complexity of mechanobiology associated with the in-vivo environment has led to the development of in-vitro systems. Cell stretching is the most common approach through the use of clinical tools such as micropipettes or tweezers (Sun et al., 2004; Nishimura et al., 2008; Sniadecki et al., 2008; Tan et al., 2010; Teitell et al., 2010). However, these techniques are not suitable for long-term experiments. Flexcell (Flexcell International Corporation), Strex Systems for cell Stretching (STREX Inc.) and ElectroForce (Bose Corporation) are the few commercially available cell stretching systems. Flexcell is one of the most sophisticated commercially available cell stretching devices (He et al., 2012; Higgins et al., 2013; Dhein et al., 2014) and the BioFlex (Flexcell International Corporation) system uses a pneumatic control to inflate or deflate elastic membrane for stretching cultured cells.

Several other custom-made devices reported in the literature utilize other approaches, such as: electromagnetic, electrostatic or optical actuation techniques. A common approach is mechanically pulling an elastic membrane using computer controlled motors (Neidlinger-Wilke et al., 1994; Arold et al., 2007; Ahmed et al., 2010). Illustrated by Shao et al. (2013) who used a controlled motor to drive a cam follower assembly, that was connected to a flexible polydimethylsiloxane (PDMS) membrane for cyclic cell stretching [17]. Micro-electromechanical systems (MEMS) integrated microactuators have also been widely used for cell stretching (Kamotani et al., 2008; Sato et al., 2010), with Deguchi et al. (2015) utilizing piezoelectric actuators to stretch the PDMS device that has integrated microchambers and membranes for cell attachment [20]. Another common technique for cell stretching is pneumatic actuation to deform a thin PDMS membrane (Huh et al., 2010; Moraes et al., 2010; Simmons et al., 2011; Mann et al., 2012), Huang Y. and Nguyen (2013) fabricated a multilayered PDMS device and used a controlled vacuum source to achieve uniaxial deformation of cells cultured on a flexible membrane [25]. However, pneumatically actuated systems may lead to uncertain experimental results 
due to air leakage and increased gas permeability into the membrane. Finally, optical trapping, shape memory alloy (SMA) actuation (Iwadate and Yumura, 2009) and dielectrophoresis (Guido et al., 2012), are other methods which have their own advantages, but these devices rarely fulfill the general requirements of cell stretching such as a high experimental throughput, well-characterised strain pattern, ease of operation, compatible with a wide range of imaging systems and biocompatibility with the cells under investigation.

The effect of strain on cell morphology and behaviour may potentially lead to improved culture conditions of olfactory ensheathing cells (OECs). In this study, OECs was of interest and the system was designed to understand the primary effect of stretching on OEC morphology and physiology. OECs are the glia cells of the primary olfactory system and are located in the olfactory nerves within the nasal cavity and the outer layer of the olfactory bulb. OECs provide support and maintenance for the olfactory sensory neurons and guide the olfactory sensory axons to their targets within the olfactory bulb (Ekberg J. A. et al., 2012). Primary sensory neurons project their dendrites to the surface of the olfactory epithelium and are directly exposed to external toxic substances and pathogens and as result frequently die. However, neural stem cells in the basal layer of the olfactory epithelium give rise to new neurons and the OECs release numerous growth factors which promote the growth of newly generated axons. The ability of OECs to aid facilitate axon growth (Windus et al., 2011) and integrate peripheral sensory axons into the olfactory bulb of the central nervous system make them prime candidates for spinal cord injury transplantation therapies (Ekberg J. A. and St John, 2014). A recent human trial has shown the potential of OEC transplantation in therapeutic and regenerative medicine for spinal cord injury (Tabakow et al., 2014; Ekberg Jenny A. K. and St John, 2015; Wang S. et al., 2016). Although the results were promising, the complexities for post transplantation survival of cells have been a major concern. Thus, better understanding of the OEC in-vivo physical environment holds the key to obtaining better post transplantation survival results using OECs.

In-vitro mechanotransduction studies can serve as a tool to better understand the functionality of OECs in their physical environment. We developed a cell stretching system that provides a heterogeneous strain pattern over a deformable membrane. The heterogeneous strain pattern obtained was utilized to observe the effect of predictable strain on OECs, and to determine the optimal strain requirement for the cell growth. The present paper reports a novel cell-stretching device based on the single sided uniaxial stretching approach using magnetic actuation. We present the mechanical design, fabrication, simulation and experimental characterisation of the device. Alongside preliminary cell culture results, which will be utilized for further optimisation of the design and the control of the device. The device allows for the active manipulation of growth and orientation of OECs in culture. 


\section{Device concept and design}

A single sided uniaxial stretching device was designed for application of strain to cultured OECs and at the same time allowing microscope access for live imaging. Figure 1 depicts the device concept and the actual cell stretching system. This system provided advantages such as ease of fabrication, replication and a heterogeneous strain pattern. The strain pattern allows for a better comparison of cell response towards different strains in the same field of view.

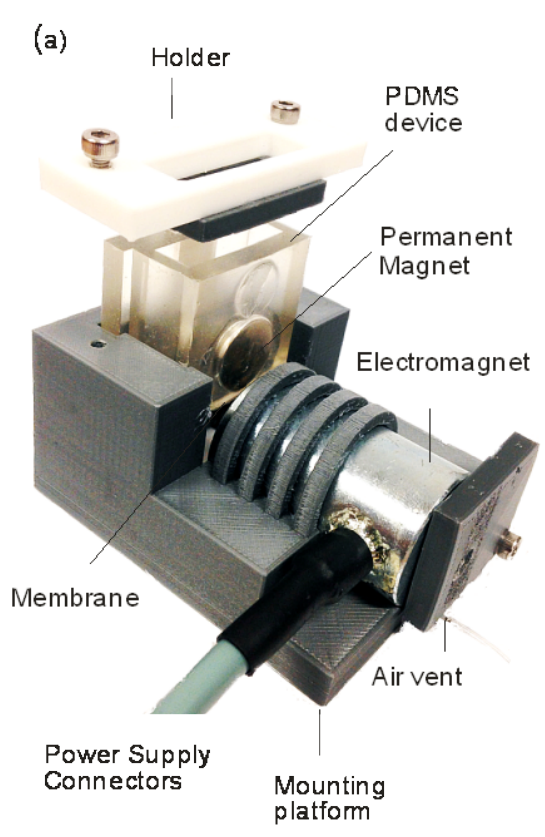

(b) OFF state Top view

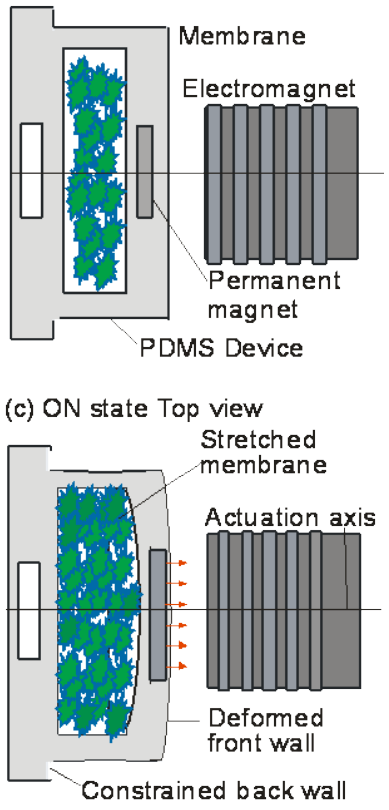

Fig. 1 The cell-stretching device: (a) the actual device; (b) the device in the relaxed state; (c) the device in the stretched state..

Figure 1a depicts the actual system for OEC stretching. The PDMS device incorporates an NdFeB permanent disc magnet with a diameter of $15 \mathrm{~mm}$ and a thickness of $2 \mathrm{~mm}$ [JL Magnet, Seoul, Korea] embedded into the front wall of the PDMS. The deformable membrane with a thickness of $200 \mu \mathrm{m}$ was bonded at the bottom of the device after a treatment with oxygen plasma. The electromagnet [JL Magnet, Seoul, Korea] is controlled by a programmable DC power supply [MK Power, Seoul, Korea] and serves as the actuator in the system. The mounting platform provides a necessary constraint to the PDMS device and also the axial alignment of the permanent magnet and the electromagnet (Fig.1b). The magnetic force generated by the embedded magnet and the electromagnet pulls the front wall, while the back wall is constrained (Fig. 1c). The unconstrained front wall is deformed as illustrated in (Fig.1c). As the membrane is bonded to the bottom of the device, the membrane is stretched uniaxially 
in the direction of the force. OECs cultured on the membrane are consequently stretched with the induced strain.

\subsection{Characterisation of the permanent magnet and the electromagnet}

The magnetic fields of the $\mathrm{NdFeB}$ disc magnet and the electromagnet were first characterised to determine the operation parameters for the device. Moreover, to design the mounting platform it was necessary to determine the acceptable minimum distance between the magnet and electromagnet in the off-state, which does not create any visible attraction between the permanent magnet and electromagnet. A gauss meter (Hirst Magnetic Instrument Ltd.) was used to measure the magnetic flux density as a function of distance and voltage for the permanent magnet and the electromagnet, respectively.

The permanent magnet was mounted onto the linear stage of a syringe pump; the gauss meter was placed perpendicular to the center axis of the permanent magnet. Reading for the magnetic flux density was taken for a distance ranging from 0 to $4 \mathrm{~cm}$ with a step of $0.1 \mathrm{~mm}$ (Fig. 2a). The results of this characterisation experiment provided a guideline for the acceptable distance between the magnet and the electromagnet, $5 \mathrm{~mm}$ was chosen for designing the mounting platform.

Furthermore, the same experimental setup was chosen for the characterisation of the electromagnet with a diameter of $25 \mathrm{~mm}$ and height of $40 \mathrm{~mm}$. The gauss meter was placed at a distance of $5 \mathrm{~mm}$ as selected from the characterisation of the permanent magnet. Readings were taken for the magnetic flux density with a voltage range of 1 to 30V. Figure $2 \mathrm{~b}$ shows that the flux density increases almost linearly with increasing voltage. A driving voltage up to $15 \mathrm{~V}$ can provide a field strength up to $50 \mathrm{mT}$ that can control the movement of the permanent magnet placed $5 \mathrm{~mm}$ apart. 

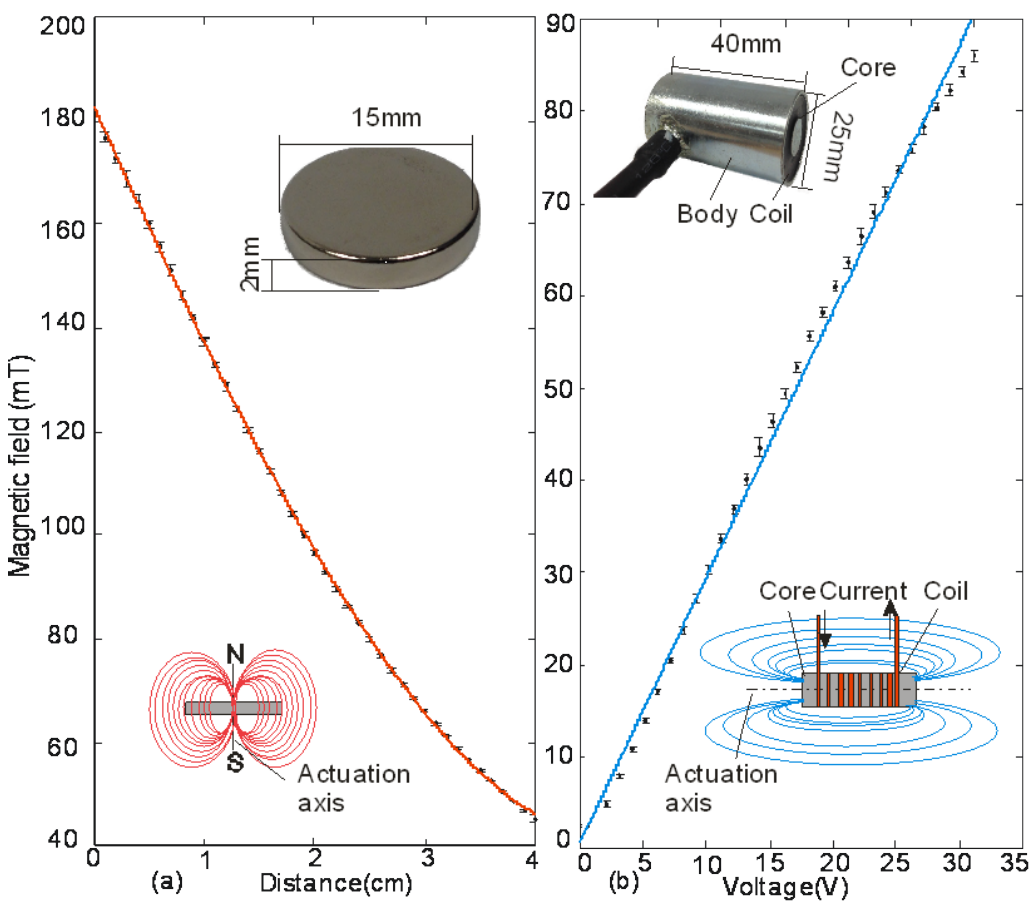

Fig. 2 Measured magnetic flux density: (a) as function of a distance from the permanent magnet; (b) as function of the voltage applied on the electromagnet.

\subsection{Design and Fabrication}

In the conceptual design phase, a simple Finite element analysis (FEA) model of PDMS device was created in COMSOL Multiphysics 4.3b to predict the device behavior and to optimise the device geometry. The location of the actuation axis along the $\mathrm{z}$ axis was the key parameter of the optimisation. Reference FEA model geometry with a total volume of 30x20x28 $\mathrm{mm}^{3}$ was built with a front wall thickness of $5 \mathrm{~mm}$, constrained wall thickness of $8 \mathrm{~mm}$, side wall thickness of $2 \mathrm{~mm}$, membrane thickness of $0.2 \mathrm{~mm}$ and the permanent magnet embedded in the font wall at $15 \mathrm{~mm}$ and $2.5 \mathrm{~mm}$ along $\mathrm{X}$ and $\mathrm{Y}$ axis from origin O. Figure 3 shows the geometry of the FEA model. Boundary conditions were implemented to constrain the back wall and all other parts were made free. An outward force of $0.5 \mathrm{~N}$ was applied to the surface magnet to represent magnetic force. The device was optimised by varying one geometry parameter while keeping other parameters constant. Using parametric sweep function, location of the magnet center along $\mathrm{Z}$ axis was varied form $8 \mathrm{~mm}$ to $20 \mathrm{~mm}$ with step of $0.5 \mathrm{~mm}$ over front wall. Figure. 3 shows the location of the permanent magnet along $\mathrm{Z}$ axis verses average displacement of membrane. The parametric study provided optimised permanent magnet position at 8.5 $\mathrm{mm}$ along $\mathrm{Z}$ axis. 


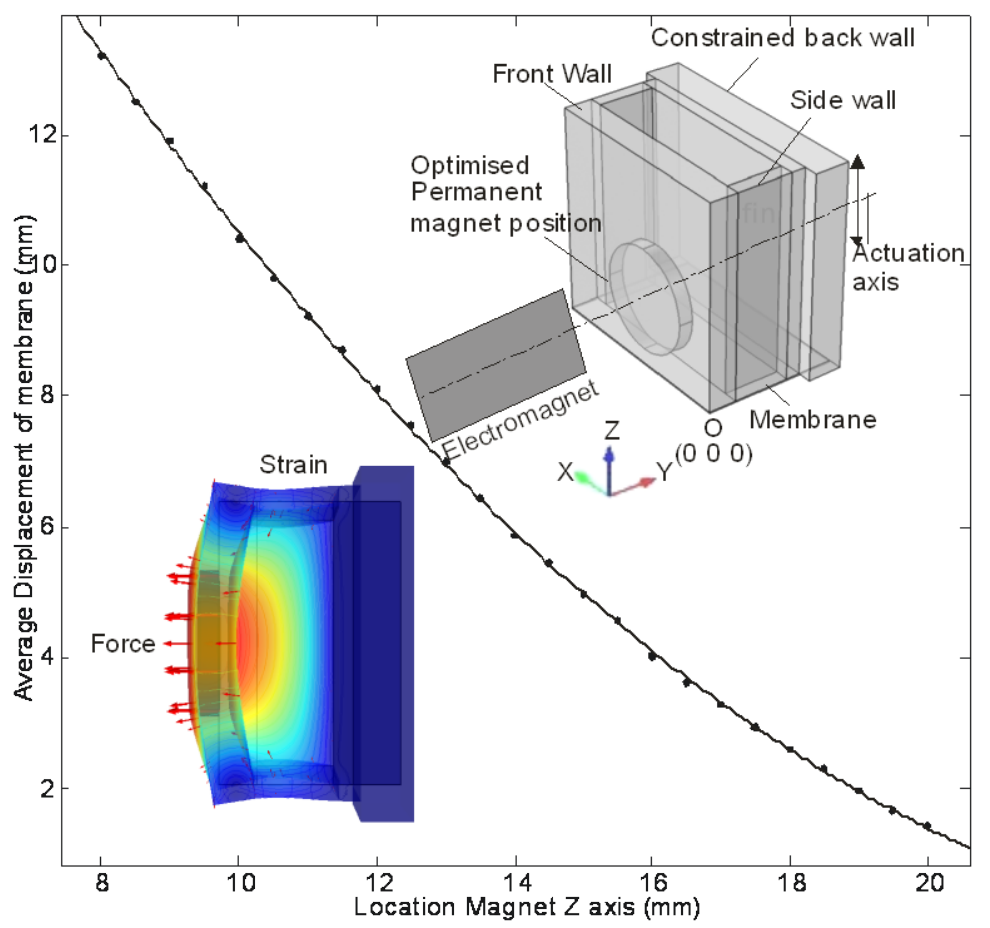

Fig. 3 Simulated results for location of actuation axis along $\mathrm{Z}$ axis verses average displacement of membrane. (Inset: Optimised geometry and simulation results of FEA model.)

The fabrication process of the cell stretching system consisted of three noteworthy steps: 1) a master mold was fabricated with aluminum by a milling machine (CSI Tech, Pyeongtaek, Korea). 2) the mounting platform and holder was made of plastics using a 3D printer (Former’s farm, Pusan, Korea) and Autodesk Inventor Professional 2015 Student Edition. 3) the PDMS device and PDMS membrane were made of PDMS (Sylgard 184 elastomer kit, Dow Corning). Fifteen gram PDMS mixture was prepared by mixing PDMS prepolymer and its cross-linker at 30:1 ratio. The mixture was poured into the master mold and degassed again for 15 minutes to remove air bubbles. The master mold was closed carefully with a cylindrical cap on mold facing inside to create a cavity for the magnet placement. After curing the master mold for two hours at $80^{\circ} \mathrm{C}$, the mold was opened and the permanent magnet was placed onto the cavity created by the cylindrical cap. To ensure the placement 10:1 PDMS and cross linker mixture was used as glue and pasted on magnet. After ensuring placement of the magnet the master mold was closed and again cured at $80{ }^{\circ} \mathrm{C}$ for 30 minutes. Replicated PDMS devices were then inspected and carefully peeled out of master mold. To prepare the deformable membrane PDMS and cross linker was mixed in 10:1 ratio. PDMS membrane was spin coated at $4000 \mathrm{rpm}$ for 2 min to achieve 0.2-mm membrane thickness and cured for two hours at $80{ }^{\circ} \mathrm{C}$ in a vacuum oven. PDMS device and membrane was cleaned with isopropanol and Deionized (DI) water and plasma bonded for 45 seconds followed by one hour curing at $80{ }^{\circ} \mathrm{C}$ in a vacuum oven to ensuring proper bonding.

\section{Device characterisation}




\subsection{Characterisation of magnetic force}

A three-dimensional (3D) finite element analysis (FEA) model of PDMS was utilized to calculate the spring constant $(\mathrm{K})$ of the experimental setup. With the known spring constant, the force generated by the embedded permanent magnet and the electromagnet was then estimated. The geometry of the device was replicated in COMSOL Multiphysics 4.3b. The Young's modulus for the device material (PDMS mixed with 30:1 volume ratio) and the membrane material (PDMS mixed with 10:1 volume ratio) was selected as 0.145 MPa and $50 \mathrm{Kpa}$, respectively (Kim et al., 2011; Palchesko et al., 2012). The back wall was fixed with the other parts able to move freely. The magnet embedded in the wall was considered as the region of interest (ROI) and displacement of the ROI was obtained by applying outwards force to the surface of the permanent magnet to mimic the magnetic force acted on it with the electromagnet turned on. The applied force was varied from 0.1 to $10 \mathrm{~N}$ to obtain the corresponding average displacement. Considering the elastic nature of the PDMS material, displacement obtained was further utilized by calculating the apparent spring constant K using Hook’s Law;

$F=-K \cdot x$

Where $F$ is the applied force in $\mathrm{N}, x$ is the displacement in $\mathrm{m}$ and $K$ is the apparent spring constant in $\mathrm{N} / \mathrm{m}$.

For experimental magnetic force calculation, we chose the same ROI as considered for the simulation and randomly marked points over ROI. The embedded permanent magnet was deflected by supplying the electromagnet with a voltage ranging from 1 to $15 \mathrm{~V}$. The displacement of the ROI was recorded using a digital camera. An algorithm was developed to calculate the displacement of the marked point from the captured images. The algorithm was implemented using Digital Image Correlation (DIC) functions and Matlab Image Processing Toolbox. Figure. 4 shows the detailed flowchart and example of the step output of the algorithm implemented in Matlab R2014a. 


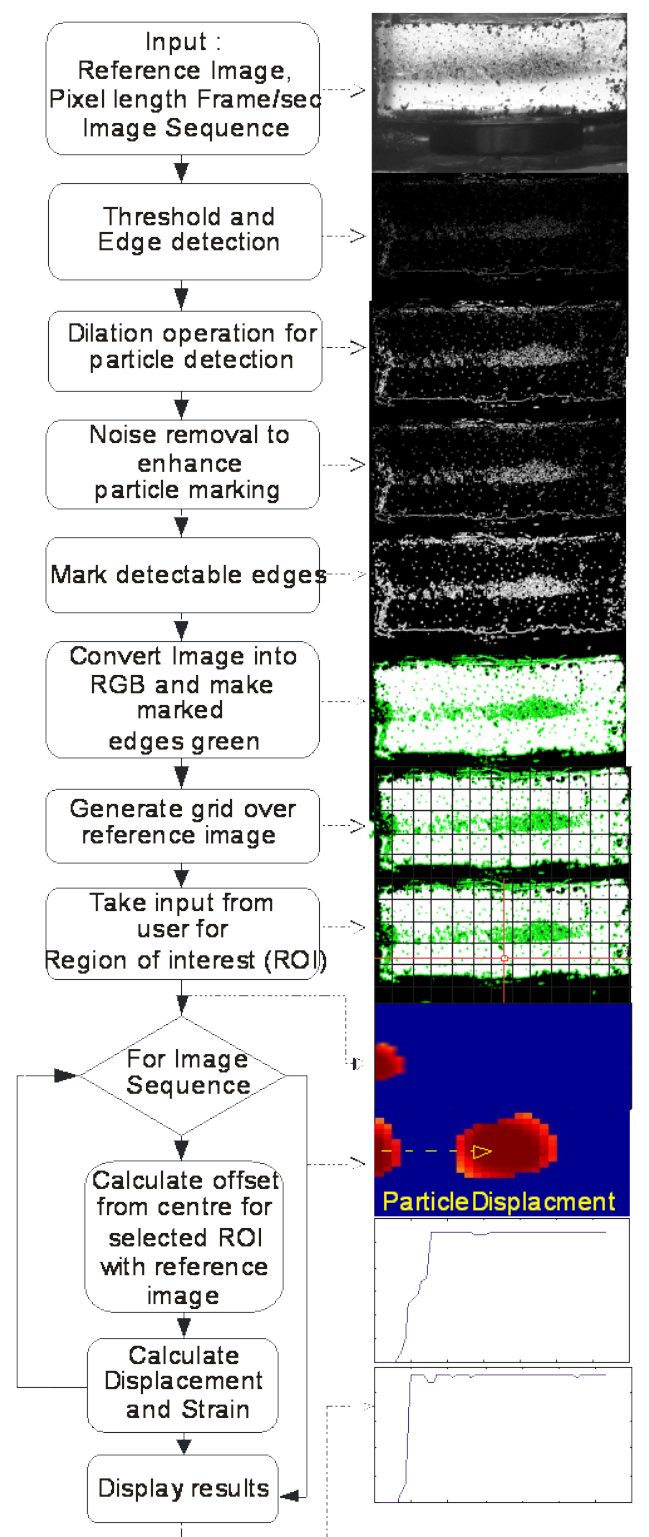

Fig.4 Flow chart for a developed algorithm to calculate displacement and strain.

An average surface displacement of ROI was calculated for each input and utilized simulated average spring constant value $(0.339 \mathrm{kN} / \mathrm{m})$ to calculate the total force generated over the surface based on Hook's law. Figure. 5 shows the experimental results of the relationship between the displacement as well as the estimated force and the applied voltage, respectively. 

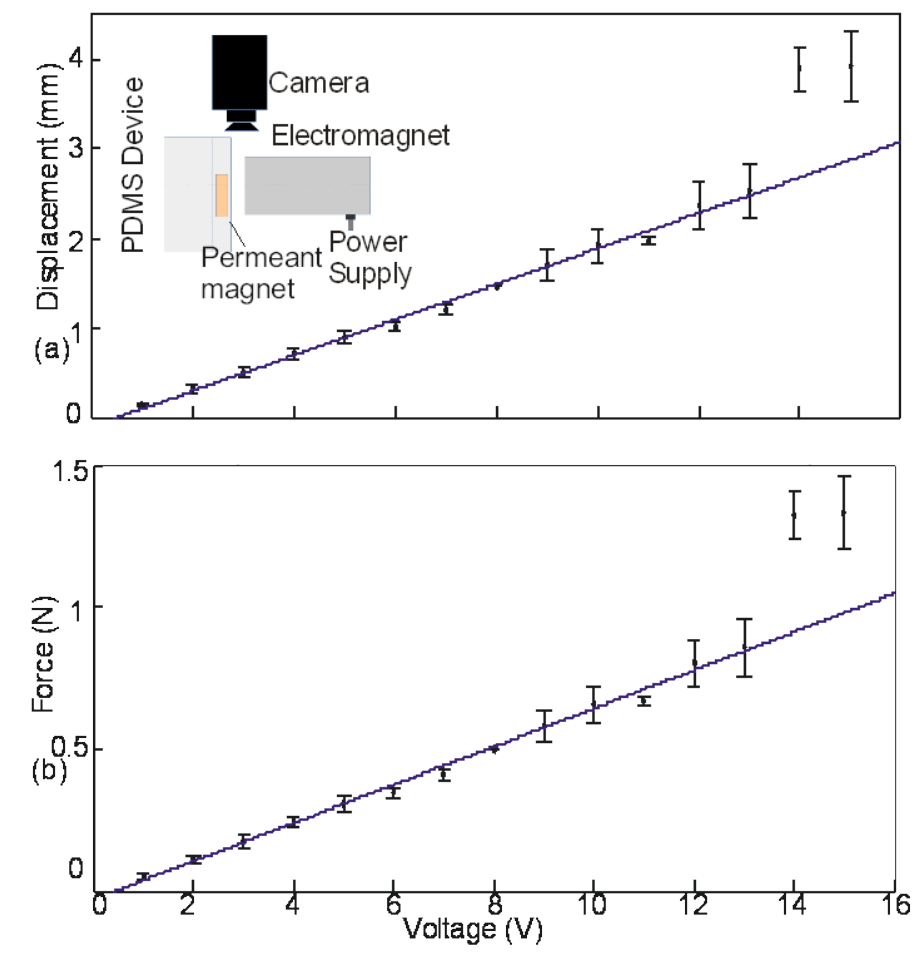

Fig. 5. Experimental characterisation of the device: (a) displacement versus voltage; (b) estimated force versus voltage.

For comparative studies, experimentally estimated force (Fig. 5b) was further utilized as an input to a comprehensive simulation model to obtain the corresponding displacement. Figure. 6 shows the simulation and experimental results.

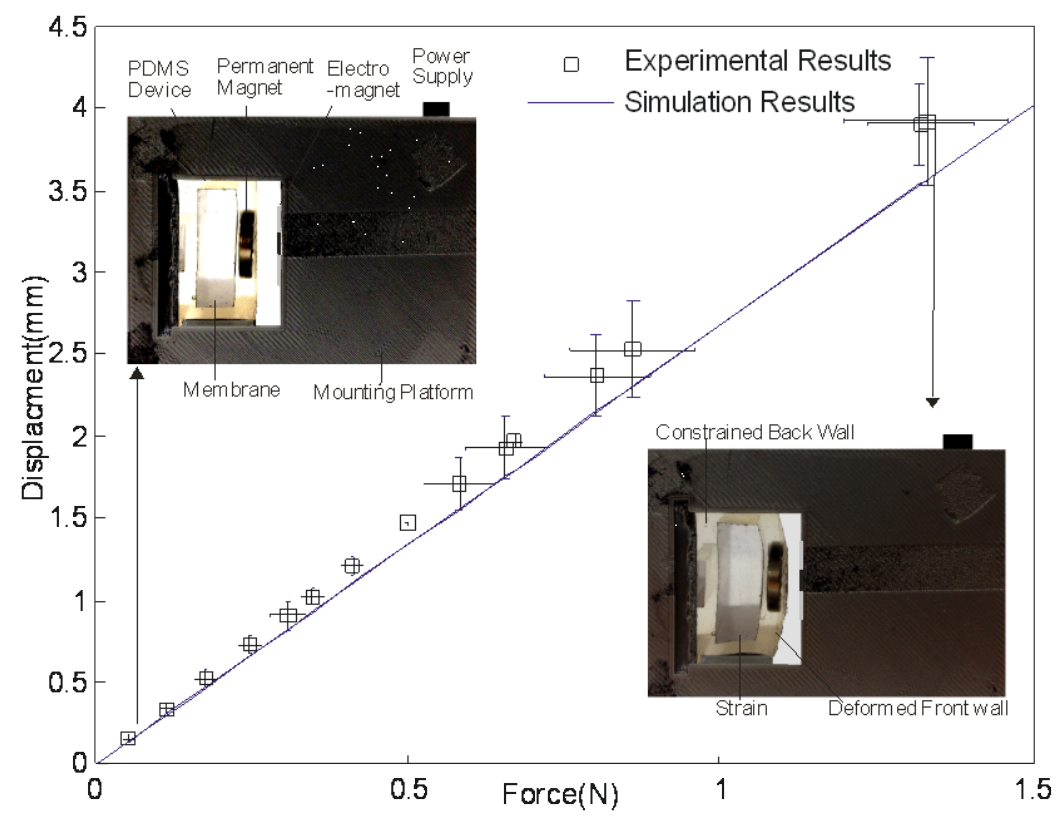

Fig. 6 Comparison of experimental and simulation force versus displacement results (Inset: Actual cell stretching system displacement due to actuation) 


\subsection{Characterisation of stretching membrane}

The numerical model was further utilized to optimise the membrane thickness. Figure. 7 shows the average strain over the membrane as a function of thickness $t$ ranging from 0.04 to $0.4 \mathrm{~mm}$ with a step of $0.04 \mathrm{~mm}$. The simulation results provided a guideline for the actual membrane thickness to be fabricated. Considering fabrication stability and suitability membrane size was fixed at $0.2 \mathrm{~mm}$.

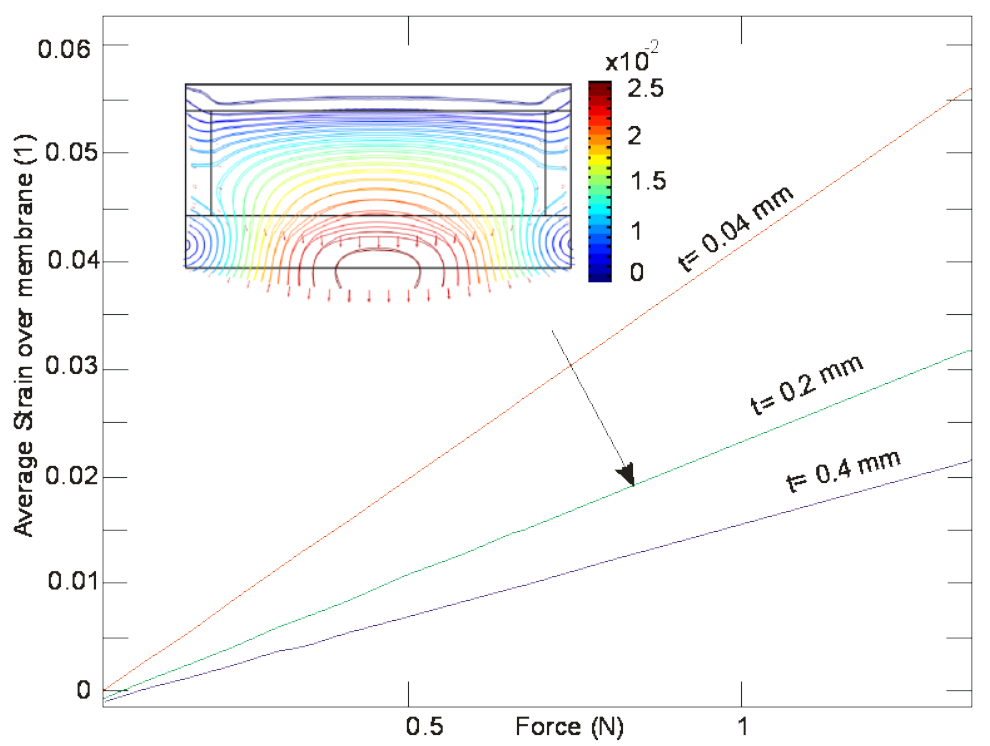

Fig. 7 Numerically simulated strain as function of appliedforce . (Inset: Strain pattern for membrnae with thickness of 0.2 $\mathrm{mm})$

\subsection{Characterisation of the strain map}

A well characterised strain map on the membrane is important to quantify the strain effect on OECs in culture. Considering the heterogeneity of the strain map whilst understanding the location of the cells with the corresponding strain are crucial for the evaluation of the cell response. The strain pattern on the membrane was investigated both numerically and experimentally.

\subsubsection{Numerical analysis}

The 3D FEA model previously used for design optimisation was further utilized to verify the experimental results. Experimentally calculated force over operation range was selected as an input to FEA model to obtain strain over the ROI. For graphical comparison, experimentally calculated force $(0.801 \mathrm{~N})$ for the $12 \mathrm{~V}$ was selected as an input to FEA model to obtain strain map over the membrane. Figure. 8a shows the simulation results. Results displayed an increasing semi elliptical pattern of strain map in the direction of force over the membrane. The central region of membrane near the deformable front wall experiences the maximum strain upon actuation while membrane region near constrained 
wall experiences the least strain. It is evident from the results that OECs in culture will experience different magnitude of strains depending on their location upon the membrane. Thus, morphological and numerical changes observed in the OECs will mainly depend on their location. For instance, cells located at maximum strain regions near the front wall are expected to have significant morphology changes. While, the region of membrane near the constrained wall will have a comparable morphology to the control cells.

Thus, cells present at any location on the membrane will experience approximately the same strain as estimated characterisation results. Also from experimental and simulation results, it was observed that the curvature of the membrane over the working range is very low allowing minimum out of plane deformation and thus does not affect real-time imaging capability of the system.

\subsubsection{Experimental analysis}

The same algorithm described in force calculation section (Fig. 4) was utilized to calculate offset displacement of the selected particle and calculate Eulerian strain. The membrane was divided into a $5 \times 12$ matrix of image regions. A minimum of three samples were taken from each region to calculate the strain pattern. Figure. 8b shows the experimental results of the strain field over the deformable membrane with actuation force generated by an applied voltage of $12 \mathrm{~V}$.

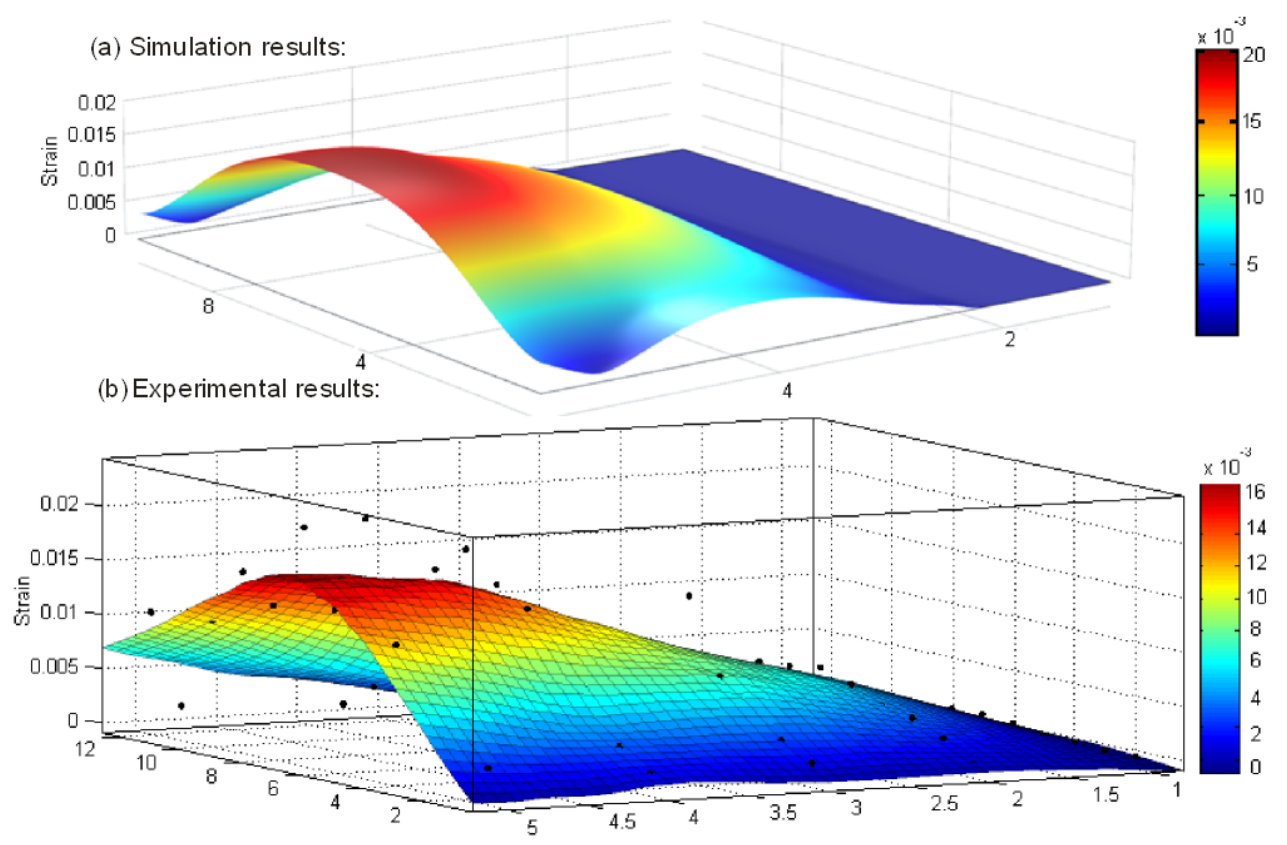

Fig. 8 Comparison of Strain map over membrane: (a) simulation; (b) experiment.

A relationship between strain over the ROI and voltage is established using same experimental platform. Figure. 9 shows the comparison between simulation and experimental results, where the 
relation between strain and voltage is linear. Thus experimental matches well to simulated results and results show consistency over the working voltage range of over the 10-15 $\mathrm{V}$ range allowing estimation of strain range over $1 \%$ to $2.5 \%$. Applied voltages of $12 \mathrm{~V}$ and $15 \mathrm{~V}$ yielded an average strain of $1.69 \%$ and $2.10 \%$, respectively.

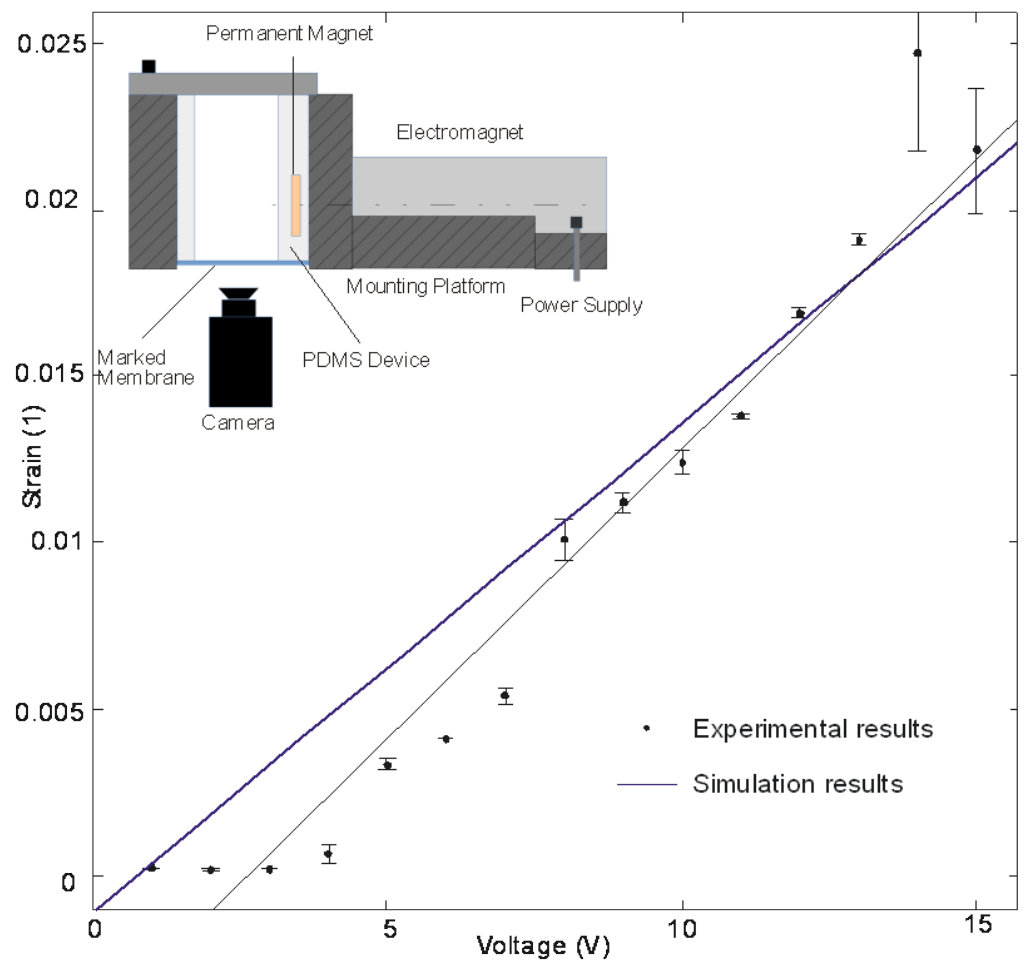

Fig. 9. Comparison of voltage versus simulation and experimental strain results at the central region of membrane. (Inset: Illustration of experimental setup).

\section{Culturing of OECs on the stretching device}

\subsection{Materials and methods}

Based on the above characterisation results, we considered the stretching condition with $15 \mathrm{~V}$ $\left(\varepsilon_{\mathrm{avg}}=2.1 \%, \varepsilon_{\max .}=2.9 \%\right)$ and a frequency $0.01 \mathrm{~Hz}$ at $50 \%$ duty cycle for the OEC stretching experiment. For OEC cultures, the device was sterilized using ethanol and dionised water followed by low-intensity UV exposure for 20 minutes. The membrane was then treated with 50 - $\mu$ l matrigel (Corning) for at least one hour, to improve OEC adhesion to the membrane.

Immortalised OECs (Tello Velasquez et al., 2014) were cultured under standard cell culture condition. DMEM/F12 culture media was enriched with FBS (10\% vol/vol), forskolin (2 $\mu \mathrm{M})$, pituitary extract (20 $\mathrm{g} \mathrm{ml}^{-1}$ ), FGF and EGF (10 ng) and gentamycin (5\% vol/vol) for the cell culture. All OECs were cultured in a humidified atmosphere of $5 \% \mathrm{CO}_{2}$ at $37{ }^{\circ} \mathrm{C}$. Cells were passaged after every 2 days at $70-75 \%$ of confluence. Seeding for the device was optimised considering various factors such as strain 
on cells, imaging capability, homogenous distribution of cells over the membrane and confluence rate (70-80\% after 24 hours). A sacking membrane deformity due to cell culture media was another factor which was a challenge for seeding optimisation. As cell culture imposed an additional load on the membrane, it affected the strain pattern and led to inconsistent results. To overcome this problem, after successive experimentation, the seeding density was optimised as 50,000 cells in a volume of 250 to $500 \mu \mathrm{l}$ of media to obtain $70-80 \%$ confluence rate after 24 hours with negligible sacking of the membrane. A relationship between lengths of the cells over the time was established to evaluate the effect of the applied strain on the cells. For imaging and evaluation purposes the membrane was divided into a 3x6 matrix of image regions. Image for each region was taken after ever four hours.

Obtained images were analyzed using ImageJ, for analysis, each image was subdivided into 5 sub-regions with 20 cells analysed from each region; the experiment was repeated three times. To calculate the average length of OECs in culture over the membrane, the length for all cells were measured. Figure.10 shows the results for the average length of the OECs with respect to time. Two distinct phases can be observed (depicted by the dashed vertical line in Fig.10) in the results, OEC growth is exponentially increased during first 8 hours and reaches a plateau phase after 10 hours.

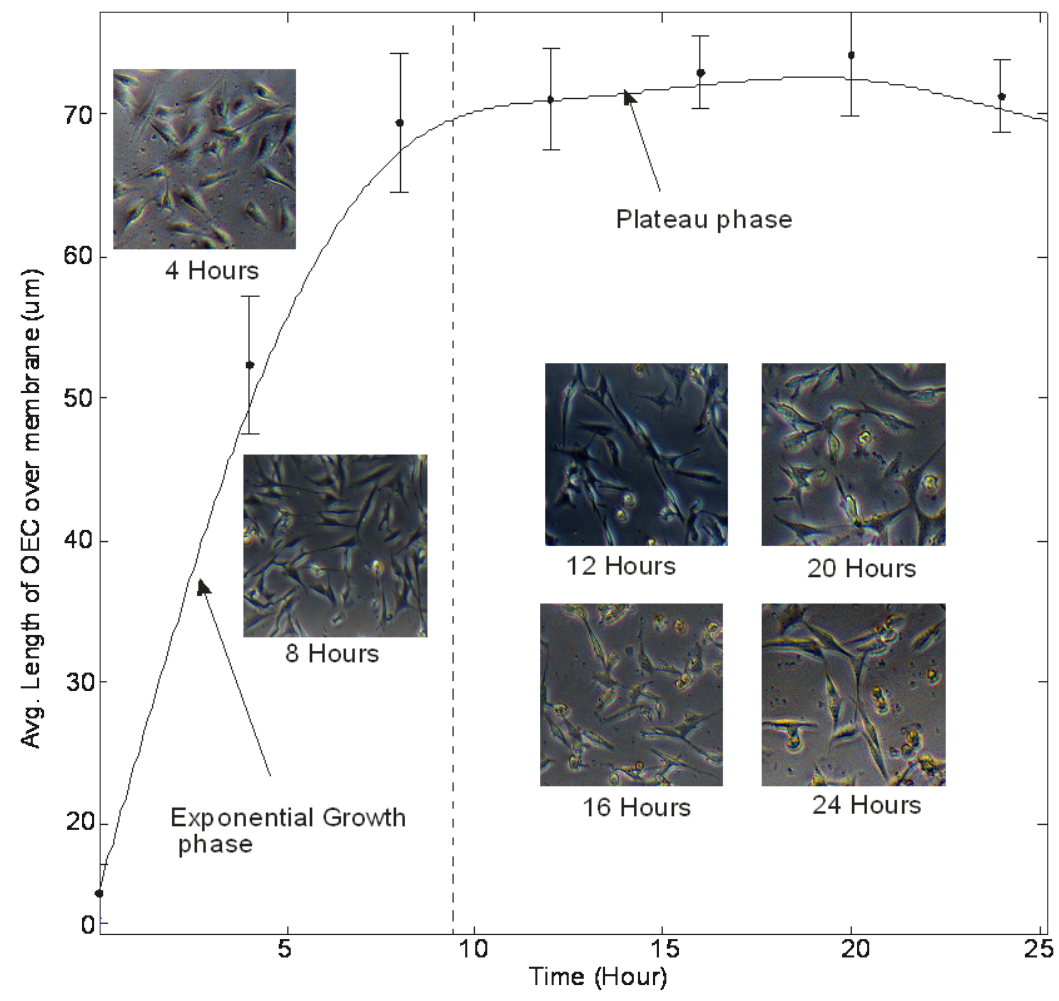

Fig. 10. Experimental results: Time verses average length of OEC over membrane. Inset: Morphology of OECs.

\subsection{Effect of strain on the OEC morphology}


Considering the results (Fig.10), 8 hours cyclic stretching experiment was designed to observe the OECs' behavior. To apply strain OECs were seeded onto the membrane with optimised seeding density (50,000 cells in $250 \mu \mathrm{l}$ of media) and allowed to attach to the membrane up to three hours. Then cyclic stretch was applied to the membrane with selected stretching parameters. The strain was transferred to the OECs and changes in morphology of OECs were observed and noted. A significant morphological change was observed in the high strain regions after four hours (Fig.11). Figure. 11 shows the length of OECs versus the degree of strain, along with OEC morphologyafter four and eight hours, along with the control (without stretching). The results show that the length of the OEC decreases linearly with the increase in strain, whilst OECs retained their morphology even after the stretching was ceased.

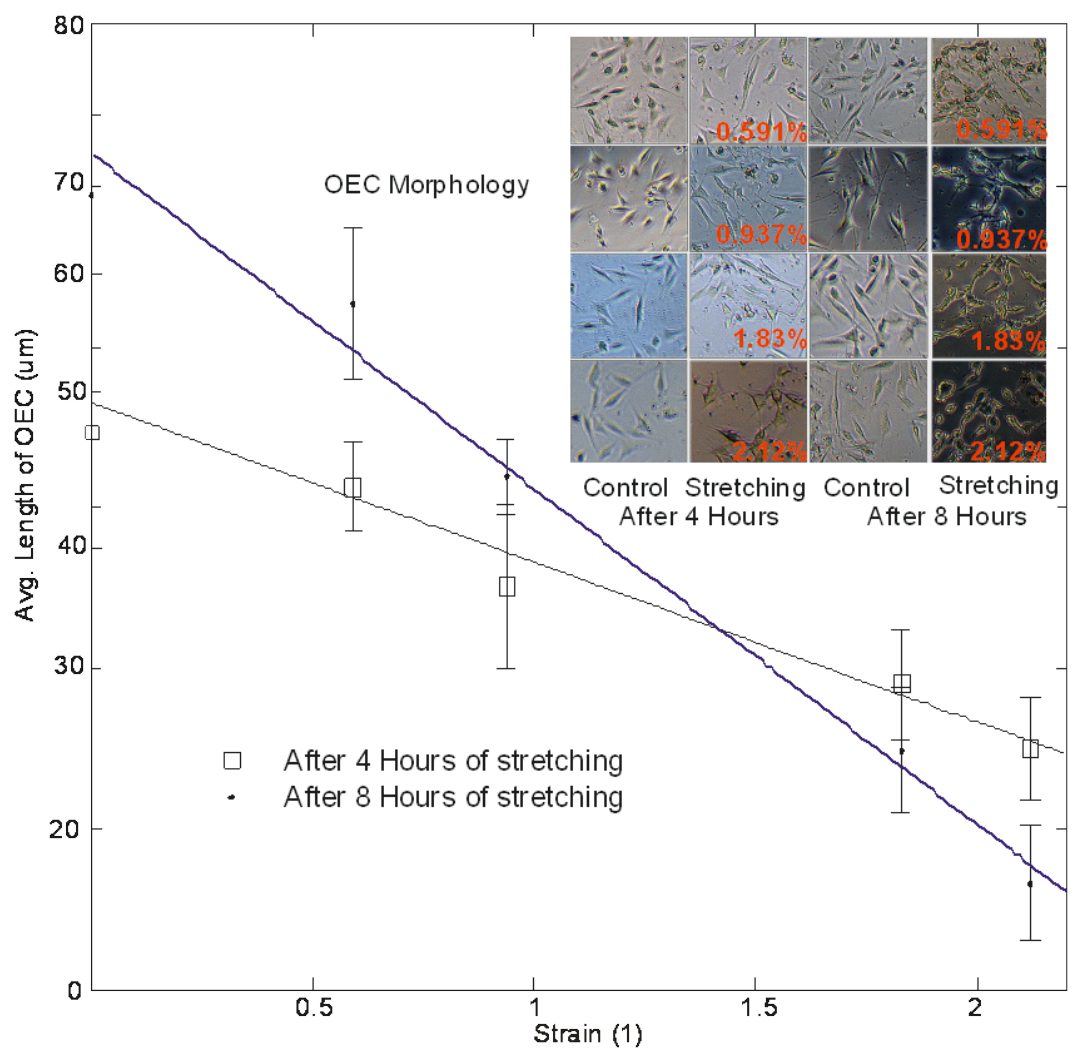

Fig.11 Experimental results: Strain verses average length of OEC after 4 hours and 8 hours. (Inset: Change in Morphology of OEC culture with and without stretching)

From the results, it is evident that the OEC morphology changes depending on the amount of strain experienced and the time subjected to the strain. Interestingly, it was observed that OECs retained their changed morphology after stretching. OECs have a plastic morphology and can rapidly alter their shape in vitro and in vivo (Windus et al., 2007; Huang Z. H. et al., 2011), in particular, OECs can extend and retract branches particularly when interacting with other cells. When co-cultured with neurons, the axons of the neurons extend along the branches of the OECs and hence increasing the length of OECs would promote axon extension (Windus et al., 2011). Our results demonstrate that increasing strain 
results in the length of the OECs decreasing suggesting that they are either are unable to maintain focal adhesions or that the altering physical relationship with the neighbouring cells disrupts the cells' ability to distinguish directional cues from growth-promoting factors within the extracellular environment. These results suggest that it is important to further understand how the changes in cell-cell interactions influence cell adhesion and growth. For example, after transplantation the constant movement of cells may decrease the successful integration of cells with the host tissue and thereby results in poorer therapeutic outcomes.

\section{Conclusions}

A device was designed, developed and characterised to apply known strain to OEC culture and investigate mechanotransduction behavior. Primary experimentations of OEC culture shows that the length OECs decreases with increase in strain experienced because of the fact that either they are unable to maintain their focal adhesion or morphology change has distrusted OECs ability to distinguished direction. The observations obtained from the experimental results illustrate the effectiveness of the developed platform and provides guidelines for the future exploration of the OECs mechanotransduction studies.

\section{Acknowledgments}

This work was supported by a grant from the Perry Cross Spinal Research Foundation grant to JSJ and BioNano Health-Guard Research Center as Global Frontier Project (H-guard 2013M3A6B2078959) through National Research Foundation (NRF) in Korea..

\section{References}

W. W. Ahmed, T. Wolfram, A. M. Goldyn, K. Bruellhoff, B. A. Rioja, M. Moller, J. P. Spatz, T. A. Saif, J. Groll and R. Kemkemer, Biomaterials, 31(2), pp. 250-8 (2010).

S. P. Arold, J. Y. Wong and B. Suki, Ann. Biomed. Eng., 35(7), pp. 1156-64 (2007).

R. P. Brandes, N. Weissmann and K. Schroder, Antioxid. Redox Signal., 20(6), pp. 887-898 (2014).

Y. Cui, F. M. Hameed, B. Yang, K. Lee, C. Q. Pan, S. Park and M. Sheetz, Nat. Commun., 6 (2015).

S. Deguchi, S. Kudo, T. S. Matsui, W. Huang and M. Sato, AIP adv., 5(6), p. 067110 (2015).

S. Dhein, A. Schreiber, S. Steinbach, D. Apel, A. Salameh, F. Schlegel, M. Kostelka, P. M. Dohmen and F. W. Mohr, Prog. Biophys. Mol. Biol., 115(2-3), pp. 93-102 (2014).

C. C. DuFort, M. J. Paszek and V. M. Weaver, Nat. Rev. Mol. Cell Biol., 12(5), pp. 308-19 (2011).

J. A. Ekberg, D. Amaya, A. Mackay-Sim and J. A. St John, Neurosignals, 20(3), pp. 147-58 (2012).

J. A. Ekberg and J. A. St John, Anat Rec (Hoboken), 297(1), pp. 121-8 (2014).

J. A. K. Ekberg and J. A. St John, Neural Regener. Res., 10(9), pp. 1395-1396 (2015).

I. Guido, C. Xiong, M. S. Jaeger and C. Duschl, Microelectron. Eng., 97, pp. 379-382 (2012).

Z. He, R. Potter, X. Li and M. Flessner, Adv. Perit. Dial., 28, pp. 2-9 (2012).

S. Higgins, J. S. Lee, L. Ha and J. Y. Lim, Biores Open Access., 2(3), pp. 212-216 (2013).

Y. Huang and N. T. Nguyen, Biomed. Microdevices, 15(6), pp. 1043-54 (2013).

Z. H. Huang, Y. Wang, X. B. Yuan and C. He, Exp Cell Res, 317(20), pp. 2823-34 (2011). 
D. Huh, B. D. Matthews, A. Mammoto, M. Montoya-Zavala, H. Y. Hsin and D. E. Ingber, Science, 328(5986), pp. 1662-8 (2010).

D. E. Ingber, Ann Med, 35(8), pp. 564-77 (2003).

Y. Iwadate and S. Yumura, Biotechniques, 47(3), pp. 757-67 (2009).

Y. Kamotani, T. Bersano-Begey, N. Kato, Y. C. Tung, D. Huh, J. W. Song and S. Takayama, Biomaterials, 29(17), pp. 264655 (2008).

D. H. Kim, N. Lu, R. Ma, Y. S. Kim, R. H. Kim, S. Wang, J. Wu, S. M. Won, H. Tao, A. Islam, K. J. Yu, T. I. Kim, R. Chowdhury, M. Ying, L. Xu, M. Li, H. J. Chung, H. Keum, M. McCormick, P. Liu, Y. W. Zhang, F. G. Omenetto, Y. Huang, T. Coleman and J. A. Rogers, Science, 333(6044), pp. 838-43 (2011).

J. M. Mann, R. H. Lam, S. Weng, Y. Sun and J. Fu, Lab Chip, 12(4), pp. 731-40 (2012).

C. Moraes, J. H. Chen, Y. Sun and C. A. Simmons, Lab Chip, 10(2), pp. 227-34 (2010).

C. Neidlinger-Wilke, H. J. Wilke and L. Claes, J. Orthop. Res., 12(1), pp. 70-8 (1994).

S. Nishimura, K. Seo, M. Nagasaki, Y. Hosoya, H. Yamashita, H. Fujita, R. Nagai and S. Sugiura, Prog. Biophys. Mol. Biol., 97(2-3), pp. 282-297 (2008).

R. N. Palchesko, L. Zhang, Y. Sun and A. W. Feinberg, PLoS One, 7(12), p. e51499 (2012).

K. Sato, S. Kamada and K. Minami, IJMS, 52(2), pp. 251-256 (2010).

Y. Shao, X. Tan, R. Novitski, M. Muqaddam, P. List, L. Williamson, J. Fu and A. P. Liu, Rev. Sci. Instrum., 84(11), p. 114304 (2013).

F. H. Silver and L. M. Siperko, Crit. Rev. Biomed. Eng., 31(4), pp. 255-331 (2003).

C. S. Simmons, J. Y. Sim, P. Baechtold, A. Gonzalez, C. Chung, N. Borghi and B. L. Pruitt, J Micromech Microeng, 21(5), pp. 54016-54025 (2011).

N. J. Sniadecki, C. M. Lamb, Y. Liu, C. S. Chen and D. H. Reich, Rev. Sci. Instrum., 79(4), p. 044302 (2008).

Y. L. Sun, Z. P. Luo, A. Fertala and K. N. An, J. Biomech., 37(11), pp. 1665-1669 (2004).

P. Tabakow, G. Raisman, W. Fortuna, M. Czyz, J. Huber, D. Li, P. Szewczyk, S. Okurowski, R. Miedzybrodzki, B. Czapiga, B. Salomon, A. Halon, Y. Li, J. Lipiec, A. Kulczyk and W. Jarmundowicz, Cell Transplant., 23(12), pp. 1631-55 (2014).

Y. H. Tan, D. Sun, J. Z. Wang and W. H. Huang, IEEE Trans. Biomed. Eng., 57(7), pp. 1816-1825 (2010).

M. A. Teitell, S. Kalim, J. Schmit and J. Reed (2010) Biomechanics of Single Cells and Cell Populations. Edited by Ho, D.

J. Tello Velasquez, M. E. Watts, M. Todorovic, L. Nazareth, E. Pastrana, J. Diaz-Nido, F. Lim, J. A. Ekberg, R. J. Quinn and J. A. St John, PLoS One, 9(10), p. e111787 (2014).

J. H. C. Wang and B. P. Thampatty, Biomechanics and modeling in mechanobiology, 5(1), pp. 1-16 (2006).

S. Wang, J. Lu, Y. A. Li, H. Zhou, W. F. Ni, X. L. Zhang, S. P. Zhu, B. B. Chen, H. Xu, X. Y. Wang, J. Xiao, H. Huang, Y. L. Chi and H. Z. Xu, Cell transplantation, 25(1), pp. 141-57 (2016).

L. C. Windus, F. Chehrehasa, K. E. Lineburg, C. Claxton, A. Mackay-Sim, B. Key and J. A. St John, Cell. Mol. Life Sci., 68(19), pp. 3233-47 (2011).

L. C. Windus, C. Claxton, C. L. Allen, B. Key and J. A. St John, Glia, 55(16), pp. 1708-19 (2007). 